2016 IEEE International Geoscience and Remote Sensing Symposium (IGARSS),

Beijing, China, 10 -15 July 2016, Pages 1404-1407

DOI:10.1109/IGARSS.2016.7729359

\title{
GEOSTARE SYSTEM PERFORMANCE ASSESSMENT METHODOLOGY
}

\author{
S. Hobbs, C. Convenevole \\ Cranfield University \\ Cranfield, Bedford, MK43 0AL, UK
}

\begin{abstract}
Geosynchronous synthetic aperture radar (GEO SAR) is attracting growing interest due to its potential for flexible and frequent imaging over continental areas. Studies are underway to evaluate mission design options and to investigate enabling technologies. This article outlines a method for assessing mission performance which accounts for actual landcover distributions and weather statistics to derive statistical estimates of mission performance relative to user-defined requirements. A technical challenge is to account accurately for the effects of surface "clutter": several methods for this are available and will be evaluated. The methodology is outlined. The performance estimates will be used to refine and validate mission design options and to build evidence for the expected benefits of GEO SAR.
\end{abstract}

Index Terms - geosynchronous, SAR, clutter, performance, statistics

\section{INTRODUCTION}

Geosynchronous radar is gathering growing interest because of its impressive potential imaging capability. The original studies were published around 1980 [1] and some of the more recent findings include work from Europe and China in particular, e.g. [2, 3, 4, 5]. However, no missions have yet flown and so many studies are being performed to try to assess its performance with increasing levels of detail and to improve the system design.

This paper presents a methodology being developing to evaluate the expected performance of GeoSTARe [6, 7]. GeoSTARe is a quasi-geostationary synthetic aperture radar mission concept being developed in Europe as a candidate for ESA's Earth Explorer programme. The baseline design is a C-band radar on a mini-satellite sited over Europe and Africa. Initial studies identified 16 applications for GeoSTARe with quantitative performance criteria specified for each. A mission design has been proposed and then the expected performance is calculated for representative scenarios (defined by target latitude and longitude, landcover class, etc.) for each application. Results from this first assessment are encouraging. In earlier studies, X-band performance exceeded

\footnotetext{
* Research sponsored by ESA
}
A. Monti Guarnieri *
G. Wadge

Politecnico di Milano
Milano, Italy

University of Reading Reading, RG6 6UR, UK

requirements in almost all cases considered and the highest priority L-band applications (atmospheric integrated water vapour) were also easily satisfied. These initial performance estimates are now being revised with the latest system design and new models of clutter effects.

The methodology presented here is intended to provide more comprehensive statistical performance estimates which account for surface cover and weather variability. The GeoSTARe imaging principle is easily implemented over static targets (e.g. urban areas) with a calm atmosphere. Images cannot be formed over water surfaces or surfaces with very low short-term coherence (e.g. tall, dense vegetation in windy conditions), since the target must remain coherent during the integration time which can be minutes to hours. Similarly, if the atmospheric refractive index change is too large and rapid then image coherence may be lost and imaging is not possible. The aim is to assess statistically the proportion of time and of target regions for which useful imaging is possible.

Section 2 outlines the methodology to be used for this study, and the datasets available are summarised in section 3. Section 4 provides a brief discussion and summarises the further work planned.

\section{PERFORMANCE ASSESSMENT METHODOLOGY}

The aim of the performance assessment is to quantify the effects of "real" weather and land-cover on the system performance. The assessment simplifies the system and uses some significant assumptions - it is not intended to be totally comprehensive. Some of the most important simplifications relate to assumptions about the phase compensation needed for image focussing. Good phase compensation depends on corrections for (a) atmospheric refractive index (ionospheric electron content and / or tropospheric humidity), (b) orbit perturbations and (c) clock synchronisation during the image integration time. In the performance assessment it is assumed that these corrections are all made satisfactorily.

Figure 1 outlines the methodology. The system is defined by parameters describing the satellite orbit relative to the target region (including slant range, orbital speed), the radar payload (transmitter power, imaging resolution, wavelength, 


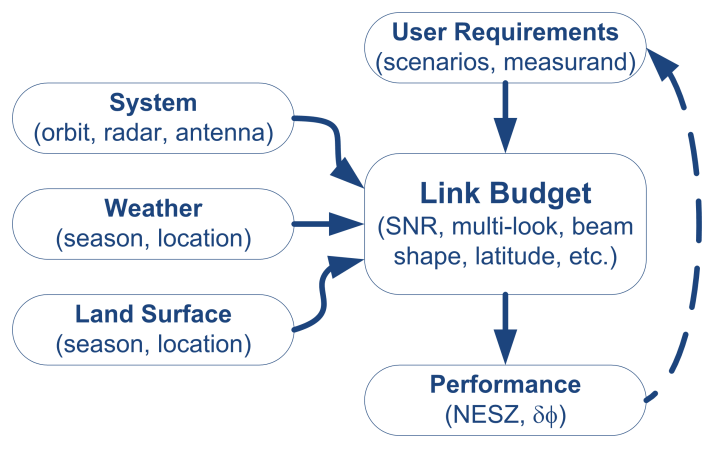

Fig. 1. Overview of methodology used to assess performance statistics

etc.) and the antenna. From these the azimuth resolution as a function of integration time can be derived. The target definition (application scenario) includes location within the beam footprint (defining incidence angle, and power relative to beam centre) and land-cover class (and hence typical surface backscatter [8]).

The user requirement defines a measurand (interferomeric phase or surface backscatter NESZ) and quantifies the measurement requirement (spatial and temporal resolution, measurand uncertainty). The "radar equation" is used to estimate the single-look SNR and thus measurement uncertainty, and then multi-looking is accounted for to estimate the measurement uncertainty at the user-defined spatial resolution. The achieved uncertainty relative to the user-requirement determines whether or not the user requirement is met.

Since the land-cover affects backscatter and surface coherence, the measurement performance is a function of landcover. We can thus estimate the fractional land-cover for which the user-defined services can be provided in various candidate imaging areas (e.g. Western Europe, North Africa).

Weather is the other main factor to be included in the performance assessment. Weather affects backscatter (e.g. backscatter difference between wet and frozen ground) and coherence. The most complicated effects are those due to coherence since low coherence means that less signal is available for interferometry and also more power is scattered due to "clutter". For GeoSTARe, the orbital speed relative to the target is so low (few $\mathrm{m} \mathrm{s}^{-1}$ ) that clutter power is spread very widely in azimuth (possibly hundreds of $\mathrm{km}$ or even well outside the beam footprint).

Several approaches to modelling clutter are available, including the work of Billingsley [9], more recent experiments performed for GeoSTARe to develop and validate revised clutter models, and direct numerical simulation of clutter due to wind-blown vegetation (using data such as [10]).

A further use of the weather statistics is to assess the variability of atmospheric conditions. This indicates how frequently "difficult" atmospheric conditions will be encountered, i.e. how often the atmosphere will prevent image

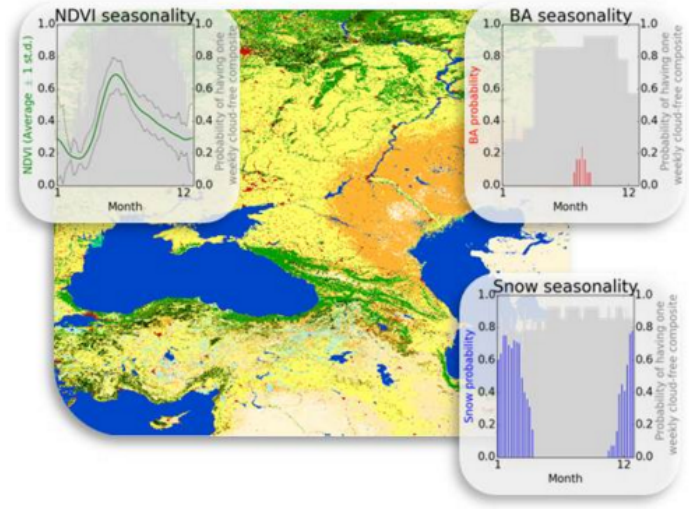

Fig. 2. Example land-cover dataset (ESA)

focussing. Short wavelengths are most sensitive to atmospheric humidity whereas long wavelengths are most affected by the ionospheric conditions. This is a secondary goal of the study and will initially be attempted only semi-quantitatively.

The final stage in the performance modelling is to use the results to improve system design. Once a quantitative method of estimating performance relative to user requirements is available, the system design parameters can be adjusted to give the best match between engineering feasibility and user requirements.

\section{WEATHER AND LAND-COVER DATASETS}

Figures 2 and 3 illustrate some of the land-cover and meteorological data available for the performance assessment. Landcover is important for many applications and so many datasets exist. The ESA dataset (from its Climate Change Initiative) shown in Figure 2 includes seasonal variations which are useful for the system performance assessments. Figure 4 outlines the methodology in more detail, showing how signal to clutter ratio (SCR) can be treated as a function of windspeed, for a given surface cover type (and landscape) and system parameters such as orbit, antenna diameter and wavelength. Windspeed statistics can then be transformed into SCR statistics, to quantify system performance in actual conditions.

Figures 5 and 6 show estimated results for an agricultural crop in southern England for several wavelengths (the SCR results assume a uniform crop cover across the whole of a broad beam footprint, and are conservative since the coherent soil backscatter is ignored). Performance statistics obtained are, for example, that the SCR will be 5 or more for $>80 \%$ of the time in L-band. This can then be expressed in terms of the service quality for users.

\section{DISCUSSION AND FURTHER WORK}

The motivation for the performance assessment is to allow us to provide quantitative estimates of system performance in re- 


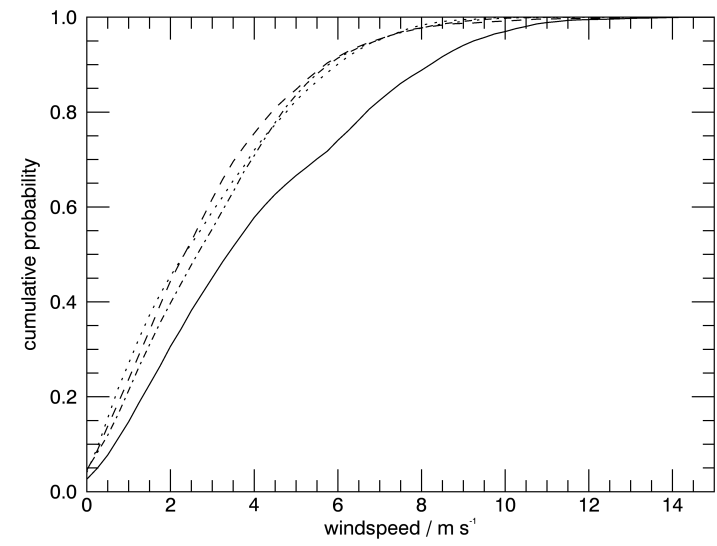

Fig. 3. Cumulative probability of $10 \mathrm{~m}$ windspeed (Cranfield, UK, for winter (solid), spring (dot), summer (dash), and autumn (dash-dot)), Dec 1996 - Nov 1997

alistic conditions. We plan to focus therefore on the most significant factors rather than a fully comprehensive study. The method builds on the first assessment of system performance (assuming single land-cover classes and ignoring weather effects), and thus can use the initial assessments for (partial) validation.

The most challenging technical aspect is the quantification of the effects of clutter. Relatively simplistic models are currently available, and only for limited conditions. The recent clutter measurements made for GeoSTARe are an important resource and further experiments are planned to give more comprehensive data and to test the models being developed. A second challenge is to quantify the effects of atmospheric structure on the ability to compensate atmospheric refractive index variations. Although algorithms have been proposed, it has not yet been possible to test them rigorously on representative scenes and thus to assess what the limits of operational phase compensation are. For this reason it is only possible to perform a semi-quantitative assessment of the influence of atmospheric conditions on system performance.

It is expected that results from this study will help to refine and validate the mission design for GeoSTARe. It is hoped that the results will provide additional evidence to justify the development of geosynchronous radar missions and that an operational mission will be in orbit within a decade.

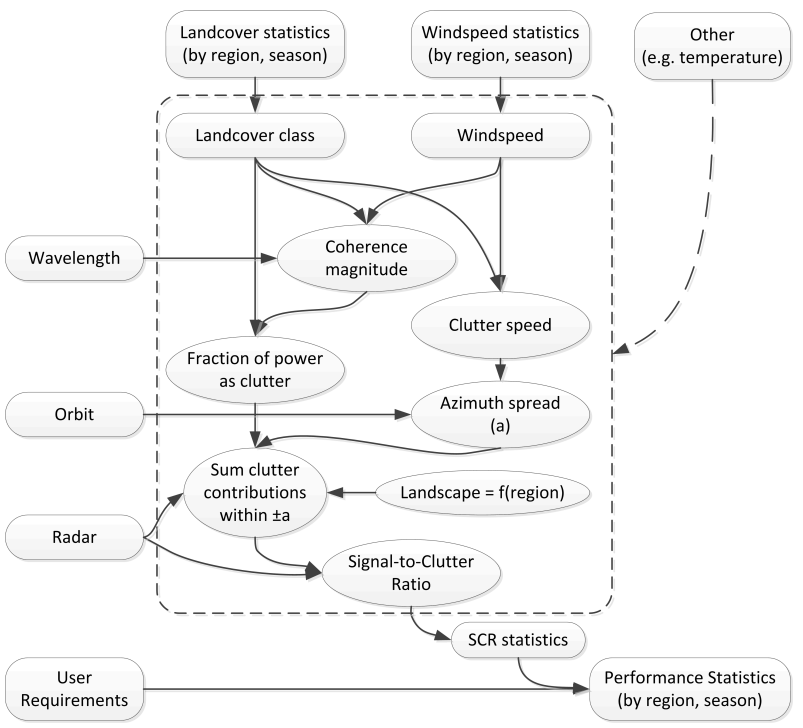

Fig. 4. Outline of the algorithm to estimate SCR as a function of windspeed (and surface cover type, region, season, etc.)

\section{REFERENCES}

[1] Kiyo Tomiyasu and Jean L. Pacelli, "Synthetic aperture radar imaging from an inclined geosynchronous orbit," 1983, vol. GE-21(3), pp. 324-329.

[2] Cheng Hu, Tao Zeng, Yu Zhu, and Zegang Ding, "The accurate resolution analysis in geosynchronous sar," in Proceedings EUSAR 2010, 2010.

[3] Stephen Hobbs, Cathryn Mitchell, Biagio Forte, Rachel Holley, Boris Snapir, and Philip Whittaker, "System design for geosynchronous synthetic aperture radar missions," IEEE Transactions on Geoscience and Remote Sensing, vol. 52, no. 12, pp. 1-14, 12 2014, doi: 10.1109/TGRS.2014.2318171.

[4] Andrea Monti Guarnieri, Antoni Broquetas, Andrea Recchia, Fabio Rocca, and Josep Ruiz-Rodon, "Advanced radar geosynchronous observation system: Argos," IEEE Geoscience and Remote Sensing Letters, vol. 12, pp. 1406-1410, 2015.

[5] Zhichao Sun, Junjie Wu, Jifang Pei, Zhongyu Li, Yulin Huang, and Jianyu Yang, "Inclined geosynchronous spaceborneairborne bistatic sar: Performance analysis and mission design," IEEE Trans. Geoscience and Remote Sensing, vol. 54, pp. 1-15, 2016.

[6] S. Hobbs, A. Monti Guarnieri, G. Wadge, and Detlef Schulz, "Geostare initial mission design," in Proc. IGARSS 2014, Quebec, July 2014, 2014.

[7] G. Wadge, A. Monti Guarnieri, S.E. Hobbs, and D. Schulz, "Potential atmospheric and terrestrial appli- 


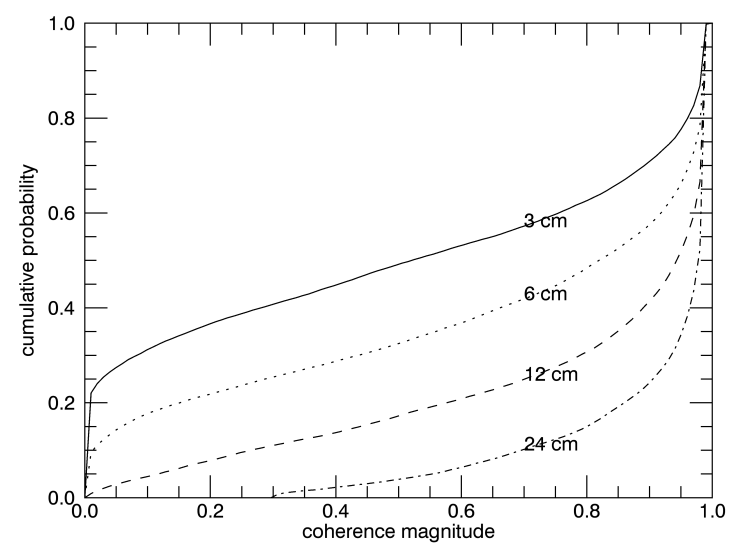

Fig. 5. Estimated cumulative probability of coherence for a wheat canopy (spring, southern England, for wavelengths of $3,6,12$ and $24 \mathrm{~cm}$, ignoring coherent ground return)

cations of a geosynchronous radar," in Proc. IGARSS 2014, Quebec, Canada, 2014.

[8] F. Ulaby and M. Dobson, Handbook of radar scattering statistics for terrain, Artech House Inc., 1989.

[9] J. Barrie Billingsley, Low-angle radar land clutter, measurements and empirical models, William Andrew Publishing Inc., USA, 2002.

[10] S.E. Hobbs, "Database of individual wheat plant motion in wind: Application to radar imaging of vegetation," Agricultural and Forest Meteorology, vol. 148, pp. 1860-1868, 2008, doi: 10.1016/j.agrformet.2008.06.009.

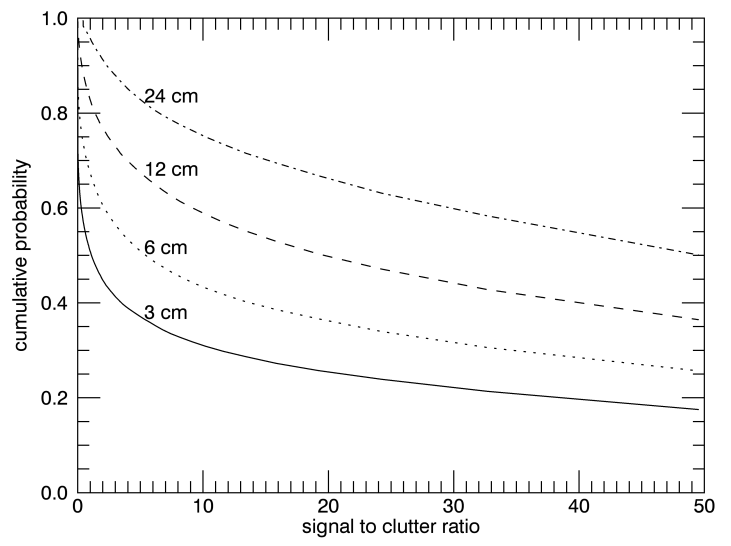

Fig. 6. Estimated cumulative probability of signal-to-clutter ratio for a wheat canopy (spring, southern England, for wavelengths of 3, 6, 12 and $24 \mathrm{~cm}$, ignoring coherent ground return) 
2016-11-03

\section{Geostare system performance assessment methodology}

Hobbs, Stephen

IEEE

Hobbs S, Convenevole C, Guarnieri AM, Wadge G, Geostare system performance assessment methodology, 2016 IEEE International Geoscience and Remote Sensing Symposium (IGARSS), Beijing, China, 10 -15 July 2016, Pages 1404-1407

http://dx.doi.org/10.1109/IGARSS.2016.7729359

Downloaded from Cranfield Library Services E-Repository 